\title{
The Salmonella Pathogenicity Island (SPI) I contributes more than SPI2 to the colonization of the chicken by Salmonella enterica
} serovar Typhimurium

\author{
Yakhya Dieye $^{\dagger 1}$, Keith Ameiss ${ }^{\dagger 1}$, Melha Mellata ${ }^{1}$ and Roy Curtiss III*1,2
}

\author{
Address: ${ }^{1}$ The Biodesign Institute, Center for Infectious Diseases and Vaccinology, Arizona State University, Tempe, Arizona 85287, USA and \\ ${ }^{2}$ School of Life Sciences, Arizona State University, Tempe, Arizona 85287, USA \\ Email: Yakhya Dieye - Yakhya.Dieye@asu.edu; Keith Ameiss - Keith.Ameiss@asu.edu; Melha Mellata - melha.mellata@asu.edu; \\ Roy Curtiss* - rcurtiss@asu.edu \\ * Corresponding author †Equal contributors
}

Published: 6 January 2009

BMC Microbiology 2009, 9:3 doi:10.1/86/147|-2180-9-3

This article is available from: http://www.biomedcentral.com/I47I-2/80/9/3

(C) 2009 Dieye et al; licensee BioMed Central Ltd.

This is an Open Access article distributed under the terms of the Creative Commons Attribution License (http://creativecommons.org/licenses/by/2.0), which permits unrestricted use, distribution, and reproduction in any medium, provided the original work is properly cited.
Received: 21 June 2008

Accepted: 6 January 2009

\begin{abstract}
Background: Salmonella enterica serovar Typhimurium (Typhimurium) is an important pathogen that infects a broad range of hosts. In humans, Typhimurium causes a gastroenteritis characterized by vomiting, diarrhea, and abdominal pains. Typhimurium infection occurs mainly through the ingestion of contaminated food including poultry, pork, eggs, and milk. Chickens that are asymptomatic carriers of Typhimurium constitute a potential reservoir for infection. The type three secretion systems encoded by Salmonella pathogenicity islands (SPI) I and 2 are major virulence factors of Salmonella. However, only a few studies have investigated their role during the infection of chickens.
\end{abstract}

Results: We have taken a mixed infection approach to study the contribution of SPII and SPI2 to the colonization of the chicken by Typhimurium. We found that SPII contributes to colonization of both the cecum and spleen in the chicken. In contrast, SPI2 contributes to colonization of the spleen but not the cecum and, in the absence of SPII, inhibits cecal colonization. Additionally, we show that the contribution of SPII in the spleen is greater than that of SPI2. These results are different from those observed during the infection of the mouse by Typhimurium where SPI2 is the major player during systemic colonization.

Conclusion: The co-infection model we used provides a sensitive assay that confirms the role of SPII and clarifies the role of SPI2 in the colonization of the chicken by Typhimurium.

\section{Background}

Salmonella enterica is a gram-negative enteric bacterium that comprises about 2500 serovars [1]. While some have a restricted host range (e.g. the serovars Typhi and Pullorum are restricted to humans and chickens, respectively), most of the $S$. enterica serovars can infect a broad range of warm-blooded animals and humans. S. enterica infects its hosts by the oral route and primarily causes two types of disease: a gastroenteritis characterized by the development of bacteria in the intestinal tract [2], and typhoid fever that results from the invasion of the systemic compartment [3]. Typhoid fever is a serious health issue in developing countries [4] but is rare in the Western world. In contrast, Salmonella gastroenteritis is an important con- 
cern worldwide. Food products, including poultry, pork, egg, and milk constitutes an important source of Salmonella infection in humans [5]. Salmonella enterica serovar Typhimurium (will be referred to hereafter as Typhimurium) is a broad host range serovar that infects humans, cattle, mice, and chickens, and is one of the major causes of food-borne human salmonellosis [6,7].

Typhimurium remains an important concern to the poultry industry [8] causing a systemic infection in newly hatched chicks, often resulting in death [9]. In older birds infection by Typhimurium leads to an asymptomatic carriage state with colonization of the digestive tract and continuous shedding $[10,11]$. These healthy carrier birds constitute a risk of contamination of newly hatched chickens, as well as the food chain leading to both important economic losses and potential harm to human consumers.

The pathogenesis of Salmonella has been extensively studied in the mouse [12]. In susceptible mice, Salmonella causes an acute systemic disease with limited intestinal manifestations [13]. Recently, a model of Salmonella enterocolitis has been developed in streptomycin-treated mice [14]. Studies using these mice and other animal models of Salmonella diseases have yielded substantial data about the molecular players involved at different levels. The Salmonella pathogenicity islands (SPIs) 1 and 2 are two major virulence determinants of S. enterica. They encode type III secretion systems (T3SS) that form syringe-like organelles on the surface of gram-negative bacteria and enable the injection of effector proteins directly into the cytosol of eukaryotic cells $[15,16]$. These effectors ultimately manipulate the cellular functions of the infected host and facilitate the progression of the infection. SPI1 and SPI2 play several roles in different organs within the host. SPI1 primarily promotes the invasion of non-phagocytic intestinal epithelial cells and the initiation of the inflammatory responses in the intestines $[17,18]$. It is also involved in the survival and persistence of Salmonella in the systemic compartment of the host [19-21]. The first characterized role of SPI 2 was its ability to promote Salmonella survival and multiplication in phagocytic cells that constitute the main reservoirs for dissemination of the bacteria into systemic organs [16]. SPI2 also plays an important role in the intestinal phase of Salmonella infection in mice $[17,22,23]$.

The regulation of SPI1 and SPI2 gene expression involves numerous transcriptional regulators located both inside and outside these pathogenicity islands. The regulation of SPI1 is particularly complex. SPI1 encodes for the five regulators HilA, HilC, HilD, InvF, and SprB (Figure 1). The first four of which are involved in regulatory pathways that lead to the activation of SPI1 genes and of genes encoding T3SS effectors located outside SPI1. In contrast to SPI1 the regulation of SPI2 genes is simpler with the SsrAB two-component system being the only transcriptional regulator encoded within SPI2 that activates the expression of SPI2 genes and of genes encoding T3SS effectors located outside SPI2. Interestingly, SPI1 regulators can regulate SPI2 genes. These include HilA that binds

\section{A $\quad \Delta s p i 1$}
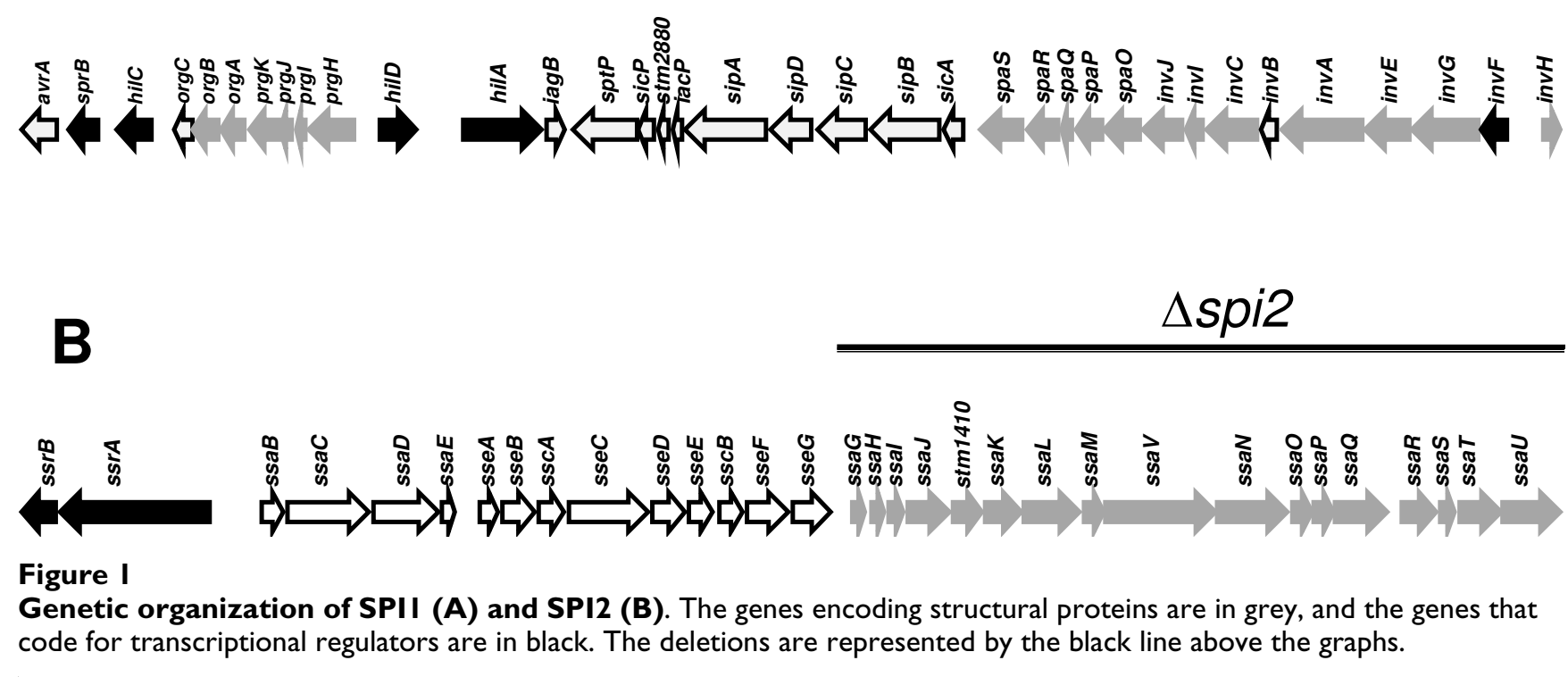
and represses the promoter of $s s a H$ [24], and HilD that binds and activates the promoter of the ssr $A B$ operon [25]. In contrast, SsrAB has never been shown to act on the expression of SPI1 genes.

Few studies have investigated the role of SPI1 and SPI2 during the infection of chickens. In studies using Typhimurium, two approaches have provided data about the roles of SPI1 and SPI2. The first approach compared colonization in chickens by infecting with single strains and enumerating colonies from internal organs. Porter and Curtiss [26] found that mutations in structural genes of the SPI1 T3SS resulted in a reduction of the colonization of the intestines in day-old chickens. Jones et al. [27] generated strains with deletions of spaS and ssaU, genes that encode structural proteins of the SPI1 and SPI2 T3SS respectively, and compared their ability to colonize the cecum and liver in one-day and one-week old chickens to that of wild type. They concluded that both SPI1 and SPI2 play major roles in both the intestinal and the systemic compartments, with SPI2 contributing more than SPI1 in both compartments. The second approach screened random transposon libraries for reduced recovery from the chicken gastrointestinal tract through cloacal swabbing. Turner et al. [28] analyzed a library of 2,800 mutants for intestinal colonization in chickens. Among the mutants that showed reduced intestinal colonization they found one in which the SPI1 gene sipC was inactivated. No mutations in SPI2 genes were identified in this screen. Morgan et al. [29] screened a library of 1,045 mutants in chickens and found two mutations in SPI1 genes and one in a SPI2 gene that led to a reduction in colonization ability. The SPI 1 mutants were unable to be recovered from $50 \%$ or $100 \%$ of the day old birds tested, while the single SPI2 gene was unable to be recovered in only $33 \%$. In this study fourteen strains with mutations in SPI1 and fifteen strains with mutations in SPI2 did not show any defect in colonization. The authors of these two studies concluded that SPI1 and SPI2 play a marginal role in the colonization of chicken intestines by Typhimurium.

To gain better insight in the role of these important virulence factors we have taken a different approach. First, we performed mixed infections in which the strains that are being compared (the wild type and a mutant, or two different mutants) are co-administered. This approach more directly addresses the contribution of SPI1 and SPI2 by decreasing the animal to animal variations inherent in such studies and giving us the ability to test the fitness of two mutants directly against each other. Second, we used one-week-old chicks that are known to be resistant to acute infection by Typhimurium [10,11] allowing us to follow the effect of the studied mutations over a relatively long period of time. Third, we used mutants in which the entire SPI 1 and/or the entire structural operon of SPI2 are deleted (Figure 1). This inactivates all the genes involved in both SPI1 and SPI2 T3SS apparatus synthesis and prevents the action of SPI1 regulators on SPI2 gene expression. Using this approach, we compared the colonization of the wild-type to that of each of the mutants.

We report here that SPI1 contributes to the colonization of both the cecum and spleen of the chicken. In contrast, SPI2 contributes to colonization of the spleen but not the cecum and, in the absence of SPI1, inhibits cecal colonization. Additionally, we show that the contribution of SPI1 in the spleen is greater than that of SPI2. These results differ from those observed during the infection of mice by Typhimurium, where SPI2 plays a major role during systemic colonization.

\section{Results}

To assess the roles of SPI1 and SPI2 in the colonization of the gut and internal organs of the chicken, we used a mixed infection approach [30]. We orally infected oneweek old chickens with mixtures of two strains. Each strain carried different antibiotic resistance markers providing a simple means of identification. At days three, seven, and fourteen post-infection, groups of chickens were euthanized. The spleen and a sample of cecum from each bird were recovered, processed and plated for enumeration of colonies as described in the Methods section. The ratio of the two strains recovered from each organ was determined and compared to the input ratio to determine the competitive index (CI, ratio of the two strains from an organ divided by the ratio in the suspension used for the infection).

\section{In Vitro Testing of SPII and SPI2 Mutants}

All strains containing SPI1 and SPI2 mutations were assayed for in vitro growth and invasion of the chicken macrophage cell line MQ-NSCU [31]. All mutants strains grew at approximately the same rate at the parent strain $\chi 4138$ (data not shown). Additionally, all mutants containing the $\Delta$ spil mutation were approximately thirty times less invasive than those with an intact SPI1 (data not shown)

\section{SPII contributes to the colonization of the cecum and of the spleen in chicken}

In chickens infected with the wild type strain and its isogenic mutant lacking the entire SPI1 $(\Delta$ spi1), the $\Delta$ spi1 cells were significantly reduced in the ceca at days three $(P$ $<0.0001)$ and fourteen $(P<0.0001)$ post-infection (Figure 2A). At day seven post-infection the difference between the two strains was not significant (Figure 2A).

Interestingly, the wild type out-competed the $\Delta$ spi1 strain in a more pronounced manner at day fourteen than at days three and seven post infection, suggesting an 

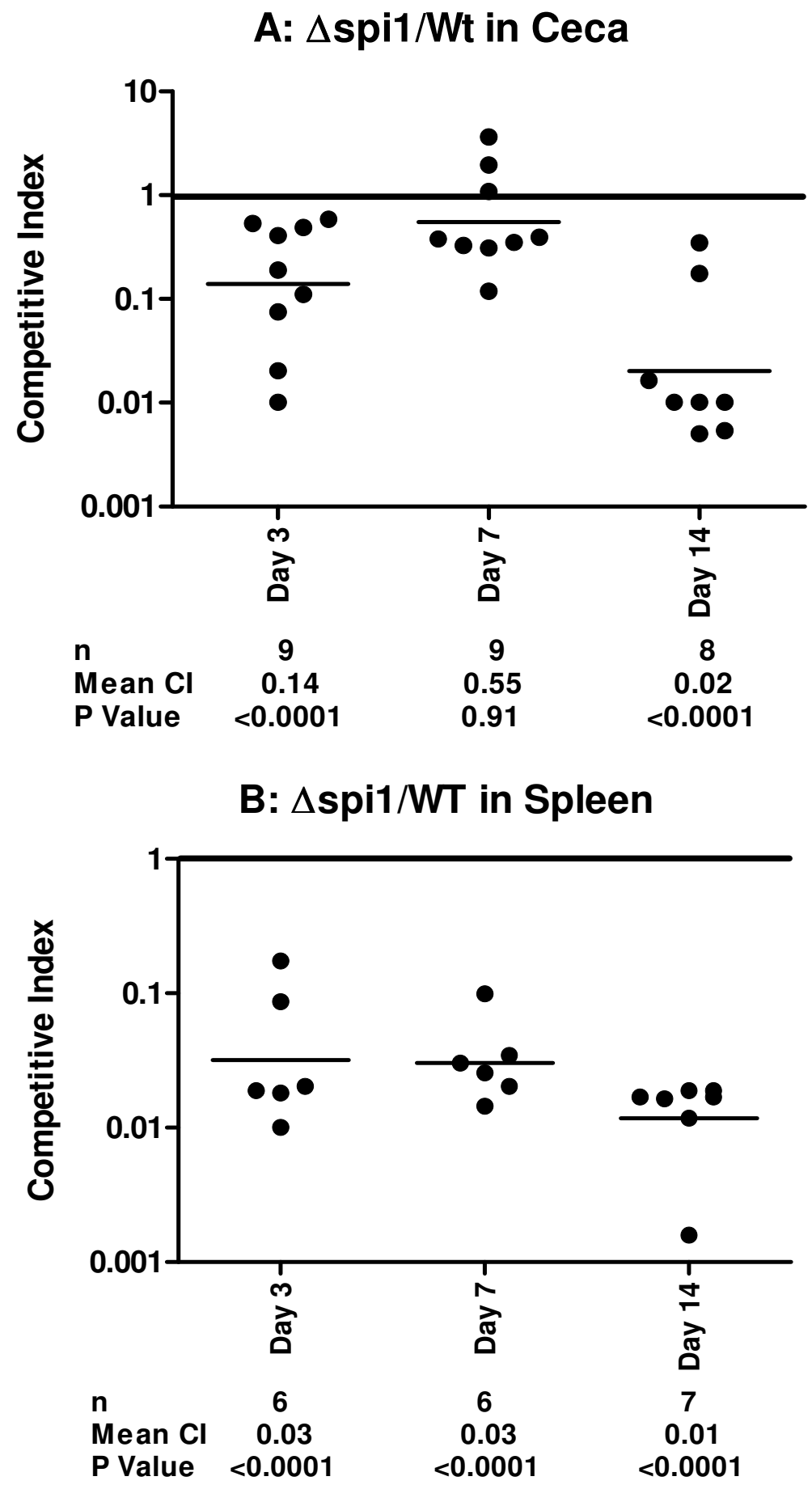

Figure 2

Contribution of SPII to the colonization of chicken cecum (A) and spleen (B) by Typhimurium. Competitive indexes are from mixed oral infections in chickens with the wild type and the $\Delta$ spil (deletion of SPII) strains. Each point represents an organ from an individual bird at the indicated day following the infection. The table summarizes the number of animals sampled $(\mathrm{n})$, the geometric mean of the competitive indexes (mean $\mathrm{Cl}$ ), and the $\mathrm{P}$ value from a two-tailed T-test. 
increased effect of the $\Delta$ spi1 mutation during long-term colonization of the cecum. For the spleen samples, the wild type out-competed the $\Delta$ spi1 strain in all the birds analyzed (Figure $2 \mathrm{~B}$ ) with the reduction of the $\Delta$ spi1 cells significant $(P<0.0001)$ at the three time points analyzed. Together these results show that SPI1 plays an important role in Typhimurium colonization of both the cecum and the spleen in chickens.

\section{SPI2 contributes to the colonization of the spleen but not of the cecum in one-week-old chickens}

In the group of chickens infected with the wild-type and its isogenic mutant lacking the T3SS of SPI2 ( $\Delta$ spi2), we did not observe significant differences, at any time point, in the cells recovered from cecal samples (Figure 3A). These results suggest that SPI2 does not contribute to the colonization of the chicken cecum by Typhimurium. To further test this hypothesis, we performed two co-infection experiments in which the effect of the $\Delta$ spi2 mutation was analyzed in the absence of SPI1. In the first experiment, we infected birds with a mixture of the wild type and the $\Delta$ spi1 $\Delta$ spi2 double mutant that lacks both SPI1 and SPI2 T3SS in order to test whether it differs from $\Delta$ spi1 with regards to the wild type.

In the second experiment, we infected the chickens with a mixture of the $\Delta$ spi1 and the $\Delta$ spi1 $\Delta$ spi2 strains in order to verify whether the phenotype observed for the $\Delta$ spi2 strain in the mixed infection with the wild type is reproducible when SPI1 is absent in the two competing strains. There was no significant difference in the cells recovered from the ceca of the chickens infected with the wild type - $\Delta$ spi1 $\Delta$ spi2 mixture (Figure $4 \mathrm{~A}$ ). This is in direct contrast with the results from the wild type- $\Delta$ spi1 mixture (Figure $2 \mathrm{~A}$ ) and both confirms that the SPI2 T3SS is not required for colonization of chicken cecum by Typhimurium and suggests that the absence of SPI2 may have a positive influence on cecal colonization. Similarly, the $\Delta$ spi1 $\Delta$ spi2 strain significantly out-competed the $\Delta$ spi1 strain in cecal samples at days three and seven post infection (Figure $5 \mathrm{~A})$. This result is in direct contrast to that obtained from the wild type- $\Delta$ spi2 infection (Figure $3 \mathrm{~A}$ ) as when both strains are SPI $1^{+}$there is no difference in cecal colonization. These results seem to suggest that the presence of the SPI2 T3SS negatively affects the colonization of the chicken cecum and that the presence of SPI1 tends to mask this phenotype. Altogether, these results both confirm that the SPI2 T3SS does not contribute to colonization of the chicken cecum by Typhimurium, and in SPI1strains actually inhibits cecal colonization.

In contrast to the observations from the cecal samples, SPI $2^{+}$strains consistently and significantly out-competed isogenic SPI2- strains in the spleen. This was observed when comparing the wild type and the $\Delta$ spi2 strain (Figure
3B), the wild type and the $\Delta$ spi1 $\Delta$ spi2 double mutant (Figure $4 \mathrm{~B}$ ), and the $\Delta$ spi1 and the $\Delta$ spi1 $\Delta$ spi2 strains (Figure $5 \mathrm{~B})$. Collectively, these results show that the SPI2 T3SS significantly contributes to the colonization of the spleen by Typhimurium in one-week-old chicks.

\section{SPII has a greater role than SPI2 in colonization of the spleen in one-week-old chicks}

Since SPI1 and SPI2 both contribute to splenic colonization and effect cecal colonization differently, we wanted to evaluate the relative importance of each of these virulence determinants. We infected chickens with a mixture of the $\Delta$ spi1 and $\Delta$ spi2 strains and found that the $\Delta$ spi2 strain significantly out-competed the $\Delta$ spil strain in the cecal samples $(P<0.0001)$ at days three, seven, and fourteen post-infection (Figure 6A). These results are consistent with the previous observation that SPI $2^{+}$cells lacking SPI1 are significantly out-competed by SPI2- bacteria (Figure $5 \mathrm{~A}$ ) and confirms that SPI1 (Figure 2A) but not SPI2 (Figures 3A, 4A, and 5A) contributes to cecal colonization.

Interestingly, the $\Delta$ spi2 strain also significantly out-competed by the $\Delta$ spil strain in the spleen at days three and fourteen post-infection (Figure $5 \mathrm{~B}$ ). This result suggests that SPI1 contributes more than SPI2 to splenic colonization. Since SPI2 has been shown in several animal models, including the mouse, to be a major factor for the survival of Salmonella in the systemic compartment of the host we decided to verify the accuracy of the results we obtained with the $\Delta$ spi2 strain in chicken spleen by performing mixed infection experiments in mice. As expected the $\Delta$ spi2 strain was out-competed by the wild type (Figure $7 \mathrm{~A}$ ) and the $\Delta$ spi1 strains (Figure $7 \mathrm{~B}$ ) in both the liver and spleen after either intra-peritoneal (Day 3) or oral (Day 5) infections. Collectively, these results show that in contrast to the mouse, SPI2 contributes less than SPI1 to splenic colonization of the chicken.

\section{Discussion}

SPI1 and SPI2 are important virulence determinants of $S$. enterica serovars that have been extensively studied in several animal models. Few studies have investigated the role of SPI1 and SPI2 in the colonization of the chicken by Typhimurium. These studies have analyzed the colonization of different organs in chickens infected with a wild type strain or with mutants of SPI1 or SPI2 in which a single T3SS structural gene was inactivated. To gain better insight in the roles played by SPI 1 and SPI 2 in the chicken we used an approach that combined mixed infections, large deletions in SPI1 and SPI2, and the tracking of infections for fourteen days. We found that SPI1 contributes to colonization of both the cecum and the spleen in chickens. In contrast, SPI2 plays a role in the colonization of the spleen, but not of the cecum. Furthermore, we show for the first time to our knowledge, that SPI1 plays a more 


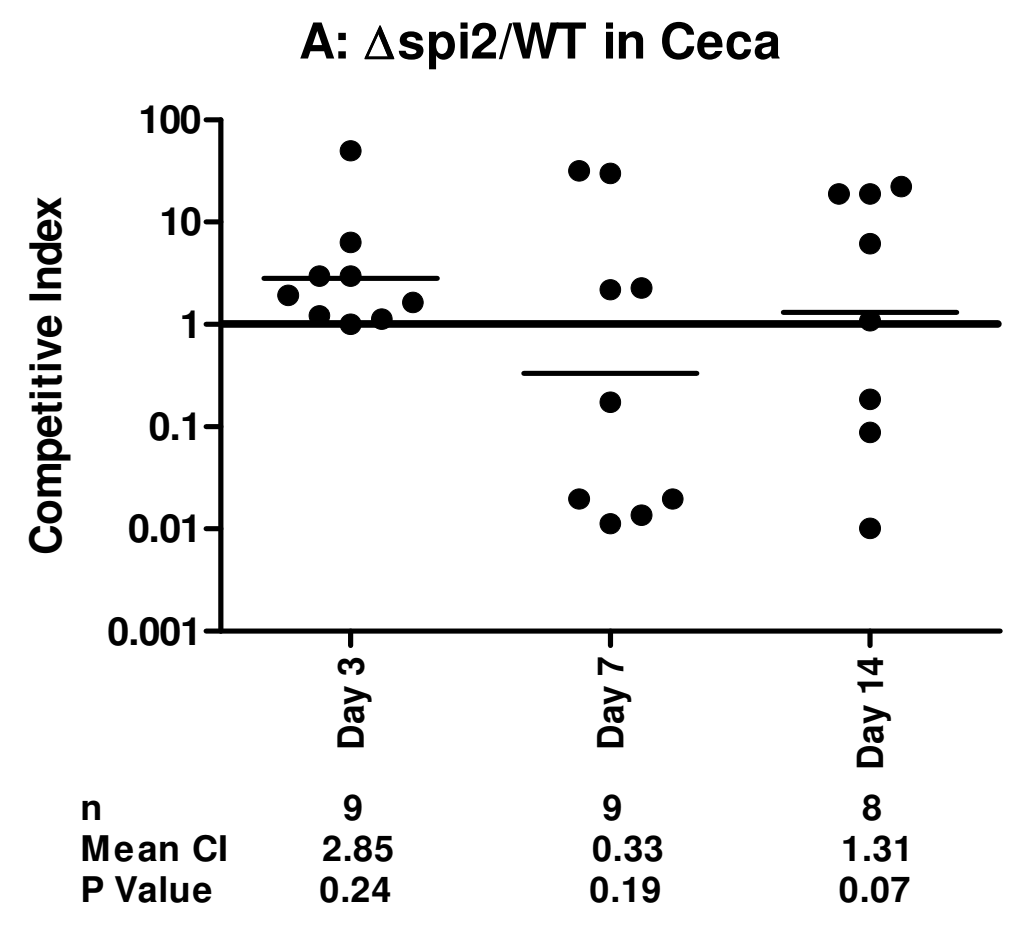

\section{B: $\Delta s p i 2 / W T$ in Spleen}

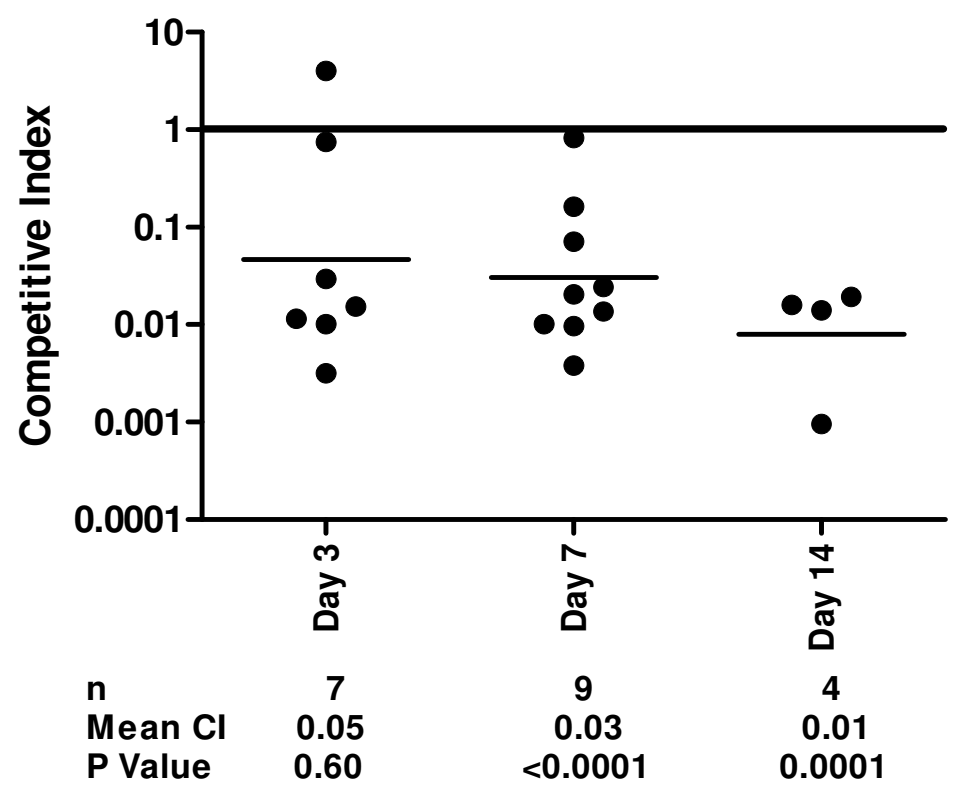

Figure 3

Effect of $\Delta$ spi2 mutation (deletion of SPI2 structural genes) in the colonization of chicken cecum (A) and spleen (B) by Typhimurium. Competitive indexes are from mixed oral infections in chickens with the wild type and the $\Delta$ spi2 strains. Each point represents an organ from an individual bird at the indicated day following the infection. The table summarizes the number of animals sampled $(n)$, the geometric mean of the competitive indexes $($ mean $\mathrm{Cl})$, and the $\mathrm{P}$ value from a two-tailed T-test. 


\section{A: $\Delta$ spi $1 \Delta$ spi2/WT - Ceca}

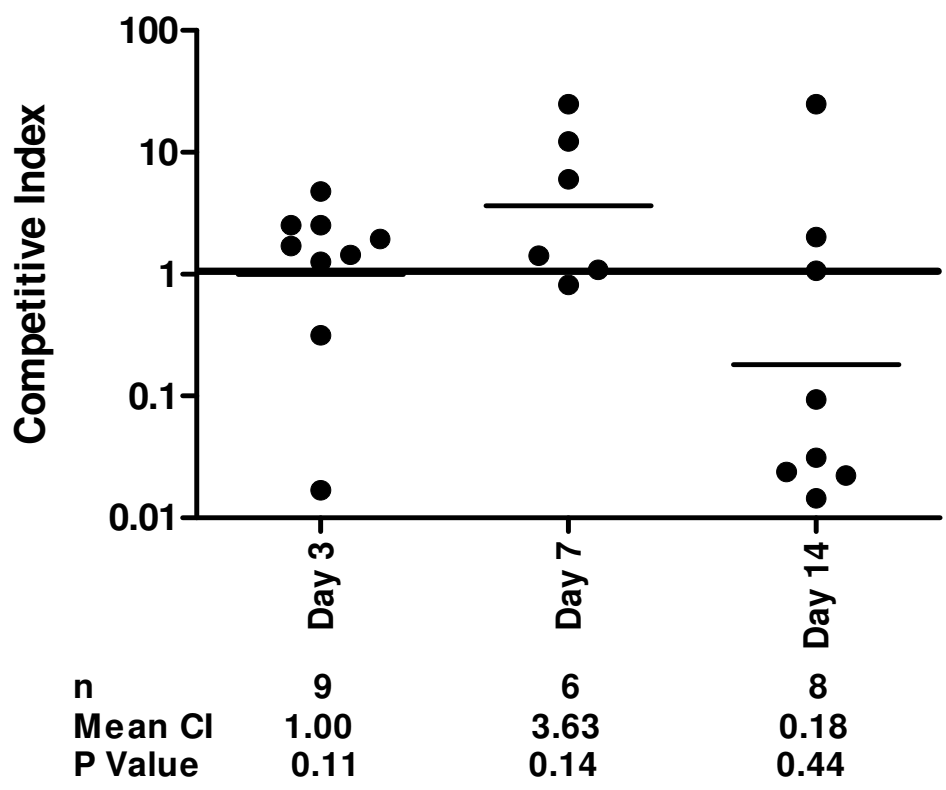

B: $\Delta$ spi1 $\Delta$ spi2/WT - Spleen

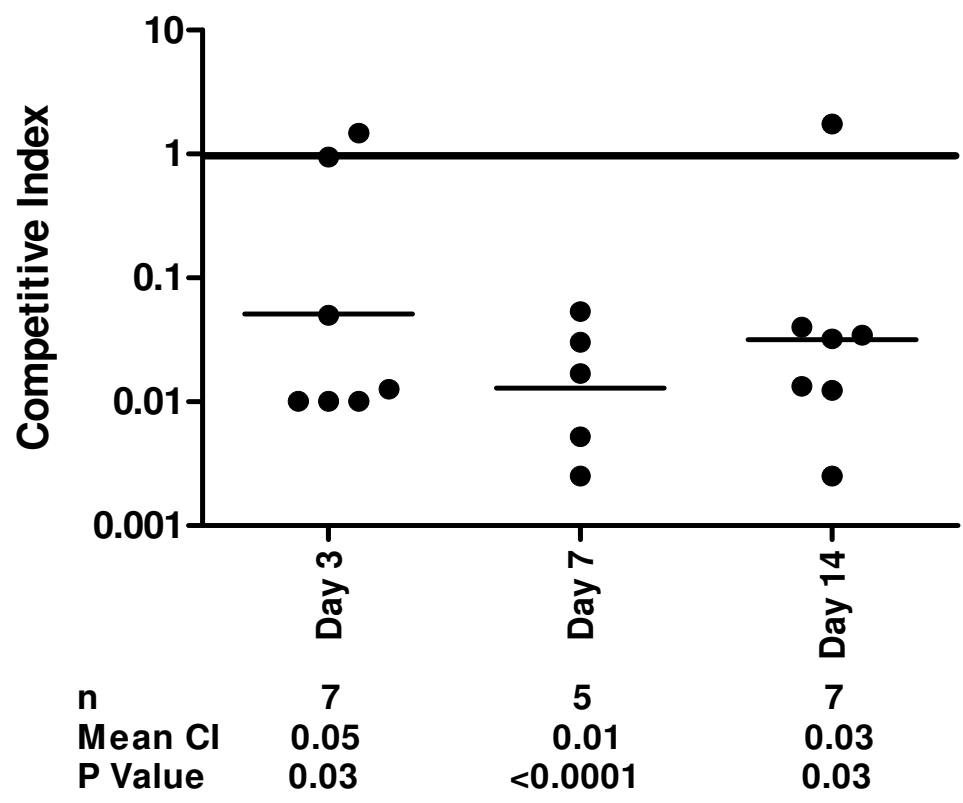

Figure 4

Comparison of wild type and $\Delta$ spil $\Delta$ spi2 (deletion of SPII and the structural SPI2 genes) colonization of the chicken cecum (A) and spleen (B). Competitive indexes are from mixed oral infections in chickens with the wild type and the $\Delta$ spil $\Delta$ spi2 strains. Each point represents an organ from an individual bird at the indicated day following the infection. The table summarizes the number of animals sampled ( $\mathrm{n}$ ), the geometric mean of the competitive indexes (mean $\mathrm{Cl}$ ), and the $\mathrm{P}$ value from a two-tailed T-test. 

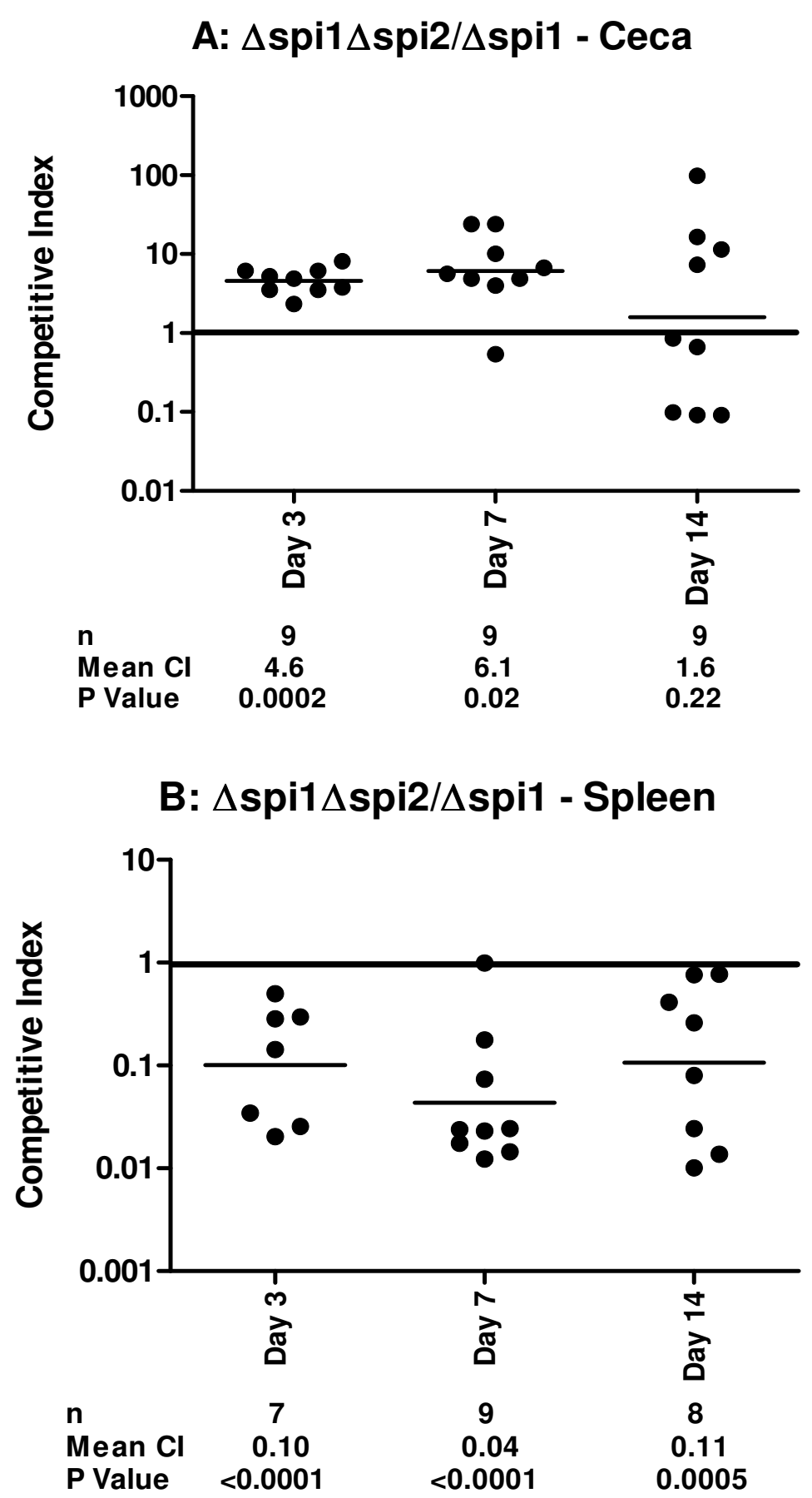

\section{Figure 5}

Comparison of $\Delta$ spil $\Delta$ spi2 (deletion of SPII and the structural SPI2 genes) and $\Delta$ spil (deletion of SPII) colonization of the chicken cecum (A) and spleen (B). Competitive indexes are from mixed oral infections in chickens with the $\Delta$ spil $\Delta$ spi2 and $\Delta$ spil strains. Each point represents an organ from an individual bird at the indicated day following the infection. The table summarizes the number of animals sampled $(\mathrm{n})$, the geometric mean of the competitive indexes (mean $\mathrm{Cl}$ ), and the $P$ value from a two-tailed T-test. 


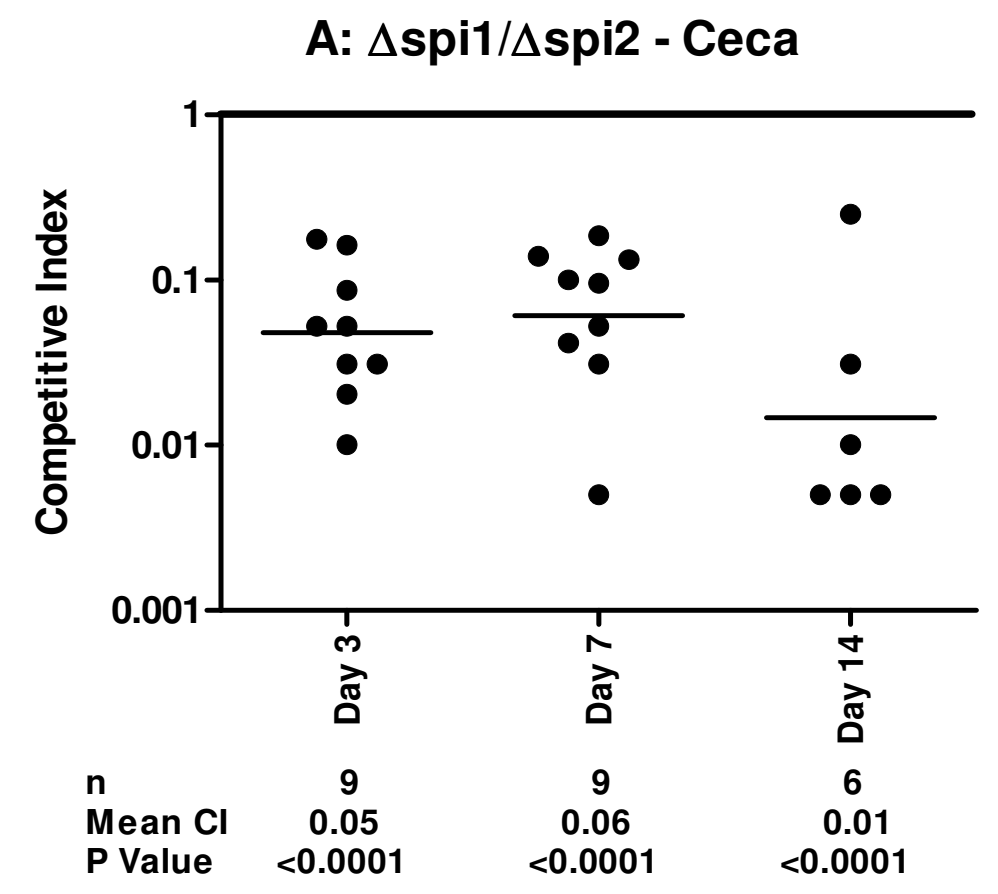

\section{B: $\Delta$ spi1/ $\Delta$ spi2 - Spleen}

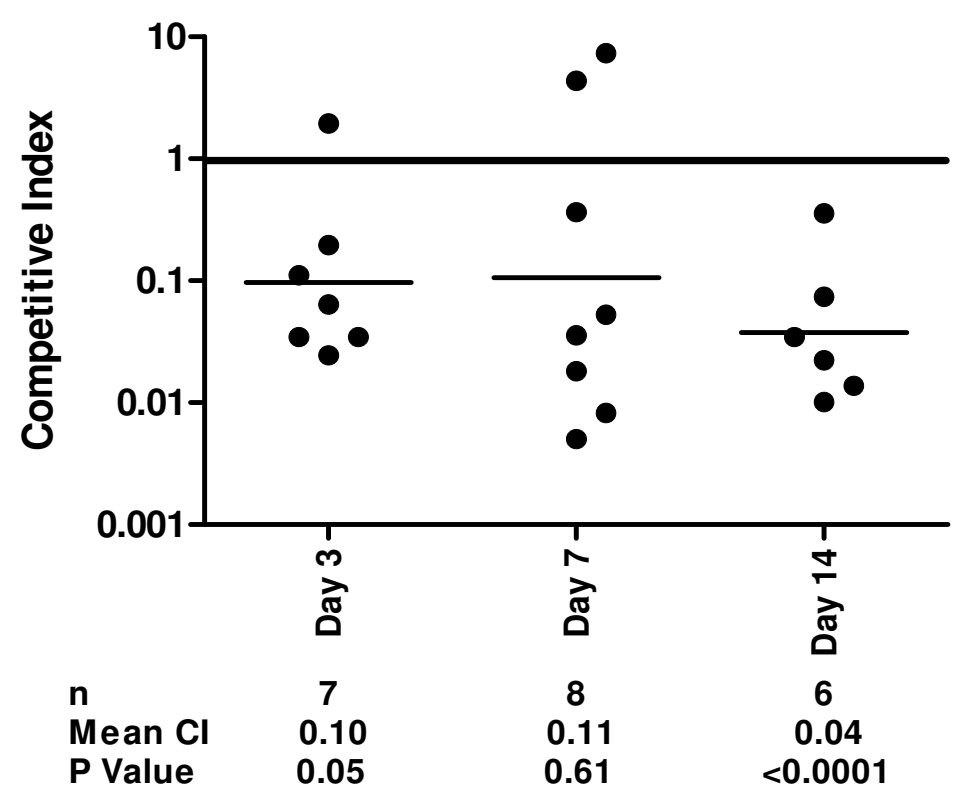

Figure 6

Comparison of $\Delta$ spil (deletion of SPII) and $\triangle$ spi2 (deletion of SPI2 structural genes) colonization of the chicken cecum (A) and spleen (B). Competitive indexes are from mixed oral infections in chickens with the $\Delta$ spil and $\Delta$ spi2 strains. Each point represents an organ from an individual bird at the indicated day following the infection. The table summarizes the number of animals sampled $(n)$, the geometric mean of the competitive indexes (mean $\mathrm{Cl})$, and the $\mathrm{P}$ value from a two-tailed T-test. 


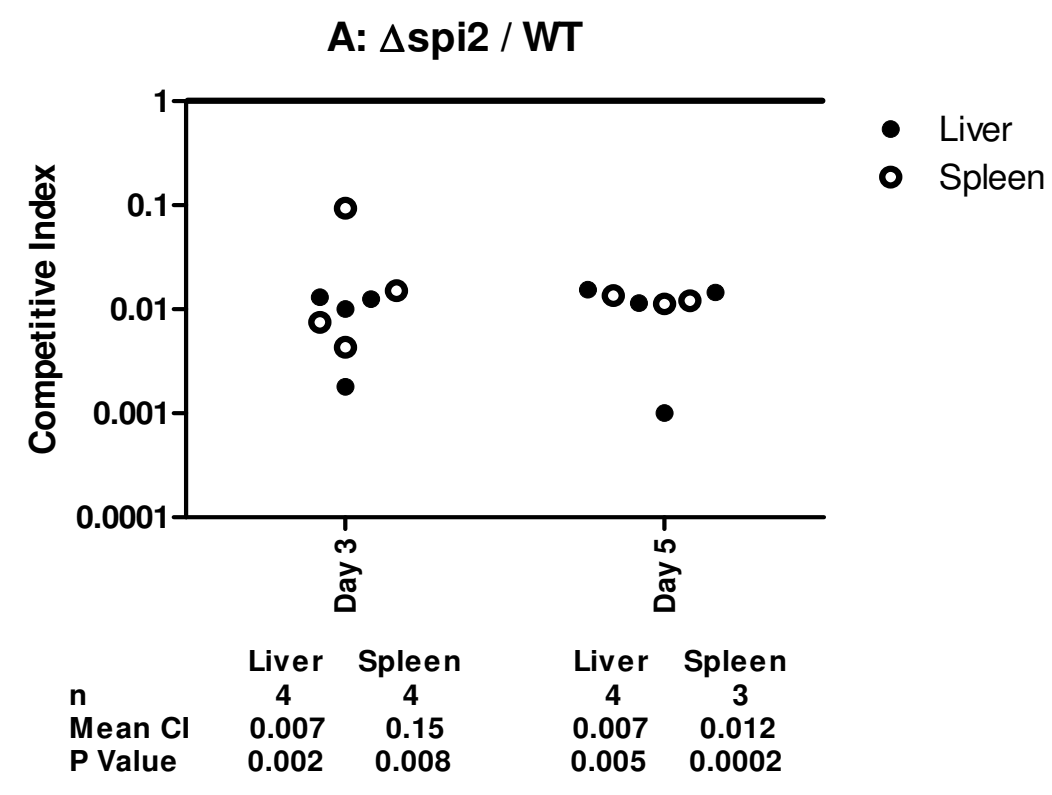

B: $\Delta$ spi $1 / \Delta$ spi2

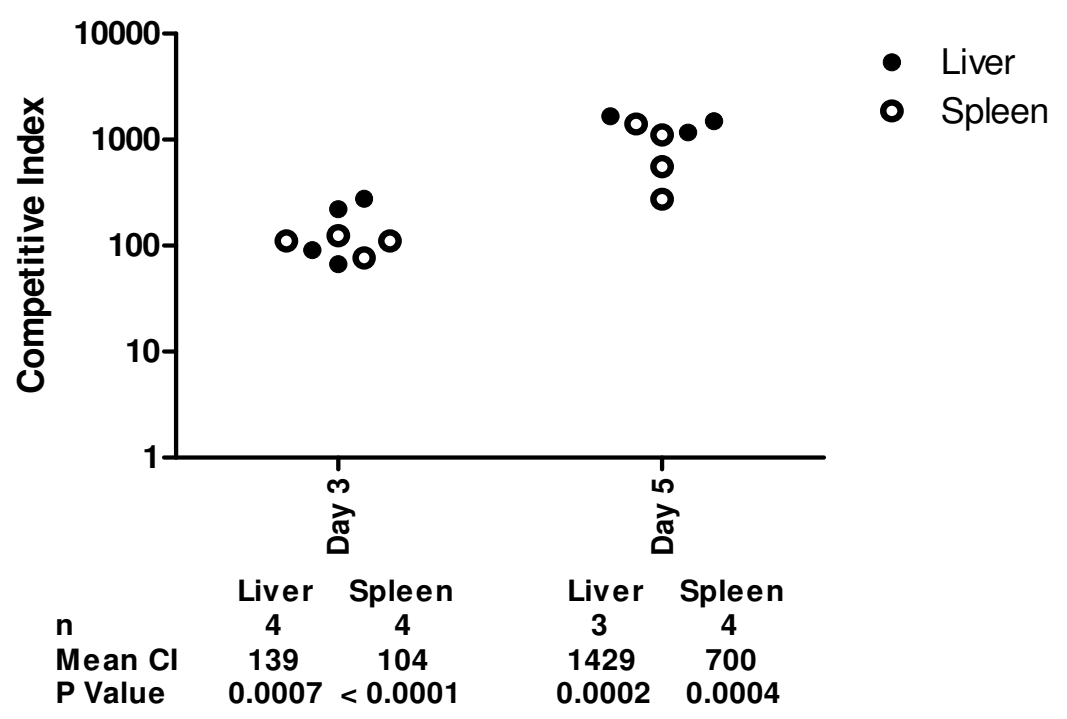

\section{Figure 7}

SPI2 is essential to the colonization of mouse spleen by Typhimurium. Competitive indexes are from mixed infections in mice with the wild type and the $\Delta$ spi2 (deletion of SPI2 structural genes), or the $\Delta$ spil (deletion of SPII) and the $\Delta$ spi2 strains. Data from day 3 and day 5 post-infection correspond to intra-peritoneal and oral infections respectively. Each point represents an organ from an individual mouse. 
important role than SPI2 in colonization of the chicken spleen by Typhimurium. We cannot exclude the possibility that the phenotypes conferred by the mutations we constructed resulted from pleiotropic effects given that we deleted the entire SPI1 and the majority of SPI2 T3SS genes, and that SPI1 and SPI2 regulators have been shown to act on the expression of genes located outside these pathogenicity islands whose functions are unrelated to T3SS [24,32]. This has been reported previously in mice where the deletion of the entire SPI1 had a different effect than a single gene deletion [33]. However, it seems unlikely as other studies have yielded results that are consistent with some of our findings. For instance, two studies that screened transposon mutant libraries of Typhimurium for reduced colonization of the chicken gastrointestinal tract either found mutations in SPI1 but not in SPI2 [28] or that SPI1 mutations had greater influence [29]. Despite the fact that cecal swabbing was used to recover strains in these two studies, which may fail to catch low level colonization, both studies still identified SPI1 as important in intestinal colonization. Cecal colonization was also reported to decrease substantially after the deletion of SPI1 T3SS components [26]. Additionally, a study with $S$. enterica serovar Enteritidis, which displays an infection pattern similar to Typhimurium, showed that deletion of the ssra gene, encoding the sensor component of the SsrAB two-component system that is the major regulator of the SPI2 gene expression, did not affect the colonization of the chicken digestive tract [34]. All together these results suggest that Typhimurium relies less on SPI2 than on SPI1 for colonization of the intestinal track in one-week-old chicks.

In contrast, Jones et al. [27] analyzed the contribution of SPI1 and SPI2 to the colonization of chickens by Typhimurium through the deletion of a single T3SS structural gene in each. They concluded that the SPI2 T3SS was required for systemic infection and played a significant role in the colonization of the gastrointestinal tract, while the SPI1 T3SS was involved in both compartments without being essential [27].

There are several important differences between that study and ours. First, Jones et al. used derivatives of the Typhimurium F98 strain [9] while we used derivatives of the UK-1 strain [36]. While both have been well characterized for virulence and persistence in chickens, their mean lethal dose $\left(\mathrm{LD}_{50}\right)$ in day of hatch chicks differ by two orders of magnitude with F98 at $5 \times 10^{5} \mathrm{cfu}$ [35] and UK1 at approximately $2 \times 10^{3}[36]$. Second, they studied mutants in which a single structural T3SS gene was inactivated while in our mutants the entire SPI1 and all the SPI2 T3SS structural genes were deleted. Third, they determined the level of colonization of the chicken by calculating the bacterial density (number of colony forming unit per gram) in the organs after administration of single strains while we infected the chickens with mixtures of the two strains being compared and determined the competitive index. These differences may account for the differences in the results.

Through the use of different combinations of mutants used for infection we have observed that strains harboring the $\Delta$ spi2 mutation have a modest advantage in the colonization of the chicken cecum, and therefore SPI2 may act to repress some factor needed for cecal colonization. However, this observation was only statistically significant when SPI1 was absent both in the strain that harbored the $\Delta$ spi2 mutation and the competing strain (Figure 5A). We have come to this conclusion based on the above observation in addition to the fact that while the $\Delta$ spi1 is out-competed by the wild type (Figure $2 \mathrm{~A}$ ), the double mutant $\Delta$ spi1 $\Delta$ spi2 is not (Figure $4 \mathrm{~A}$ ). We do not know the basis of this disadvantage conferred by the presence of SPI2 in the colonization of chicken cecum by Typhimurium. One explanation is that genes deleted from SPI2 may normally act to repress some factor needed for the colonization of the cecum but in their absence this factor is not repressed, thus increasing invasion. An alternative explanation may be that the phenotype conferred by the $\Delta$ spi2 mutation in not decreasing intestinal colonization results from the absence of SPI1 regulators, such as HilD, that are known to regulate SPI2 genes, including the SsrAB central regulator. Additional investigations are needed to test these hypotheses.

In contrast to what we have observed in chickens, SPI2 is the major contributor for spleen colonization in BALB/c mice. The infection by Typhimurium in these two animal models leads to different outcomes. In mice, Typhimurium causes an acute systemic infection, frequently resulting in death, while in one-week or older chickens, the infection leads to heavy colonization of the intestinal track and asymptomatic carriage. It is interesting to note that in animal models where Salmonella infection results in acute systemic disease, SPI2 is a major player in the systemic infection. These include the infection of mice by Typhimurium [12], and the systemic disease in chickens infected by serovars Pullorum [37] and Gallinarum [38]. In contrast, in animals where infection results in healthy carriage, such as in chickens, SPI2 plays a minor role in the persistence of the bacteria in the systemic compartment. This is demonstrated in the present study, and has been reported for Typhimurium in pigs [39], and for serovar Enteritidis in chicken [40]. This difference in contribution of SPI2 in these two situations indicates that SPI2 is an important factor of Salmonella host specificity. 


\section{Conclusion}

We have taken a mixed infection approach to study the role of SPI1 and SPI2 in the colonization of the chicken by Typhimurium. We confirmed the contribution of SPI1 to the colonization of both the cecum and the spleen, and showed that SPI2 is involved in the colonization of the spleen but not of the cecum and, may have a negative effect on cecal colonization. Additionally, we show that SPI1 plays a greater role than SPI2 in the colonization of the spleen in chickens. In contrast, SPI2 is more important than SPI 1 for systemic colonization in mice. The approach we used in this study constitutes a sensitive assay that provided new insights into the role of SPI1 and SPI2 during infection.

\section{Methods}

Bacterial growth, enzymes, reagents, and transduction

The bacterial strains were grown in LB broth [41] or on LB plates at $37^{\circ} \mathrm{C}$. The following antibiotics were obtained from Sigma and used at the following concentrations when required: kanamycin $(\mathrm{Km}), 50 \mu \mathrm{g} / \mathrm{ml}$, ampicillin, $100 \mu \mathrm{g} / \mathrm{ml}$, chloramphenicol (Cm), $20 \mu \mathrm{g} / \mathrm{ml}$, nalidixic acid (Nal), $30 \mu \mathrm{g} / \mathrm{ml}$.

General molecular biology techniques were performed essentially as described [42]. Restriction and modification enzymes were purchased from Invitrogen (Carlsbad, CA) or New England Biolabs (Beverly, MA), and used as recommended by the manufacturers. PCR primers were purchased from IDT Inc. (Coralville, IA). P22 transduction was performed as described [43].

\section{Strains}

The following Typhimurium strains, that are derivatives of the UK-1 wild-type strain, were constructed and used in this study. (I) The SPI1+SPI2+ strain $\chi 4138$, gyrA1816,

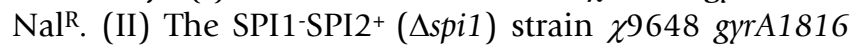
$\Delta\left(\right.$ avrA-invH)-2::cat, $\mathrm{Nal}^{\mathrm{R}}, \mathrm{Cm}^{\mathrm{R}}$. (III) The SPI1+SPI2-

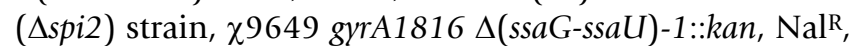
$\mathrm{Km}^{\mathrm{R}}$. (IV) The SPI1-SPI2- ( $\Delta$ spi1 $\Delta$ spi2) strain $\chi 9650$ gyrA1816 $\Delta\left(\right.$ avrA-invH)-2::cat $\Delta($ ssaG-ssaU $)-1:: k a n, \mathrm{Nal}^{\mathrm{R}}$, $\mathrm{Cm}^{\mathrm{R}}, \mathrm{Km}^{\mathrm{R}}$.

\section{Strain construction}

The $\chi 4138$ strain was made by P22-mediated transduction of the gyrA mutation from $\chi 3147$ [44] into the wildtype UK-1 strain $\chi 3761$, selecting for nalidixic acid resistance.

The mutations in SPI1 and SPI2 were constructed in strain JS246 [45] using the $\lambda$-red recombination system [46]. The deletion of the T3SS genes of SPI1 was performed using a PCR fragment obtained with the primers YD142 (5'gctggaaggatttcctctggcaggcaaccttataatttcagtgtaggctggagc tgcttc3') and YD143 (5'taattatatcatgatgagttcagccaacggtgat atggcccatatgaatatcctccttag3'). YD142 harbors 40 nucleotides that bind downstream of the stop codon of the avrA gene, and 20 nucleotides (in bold) that correspond to PS1 [46]. YD143 harbors 40 nucleotides that bind downstream of the invH gene, and 20 nucleotides (in bold) that correspond to PS2 [46]. The T3SS2 structural genes of SPI2 were deleted using a PCR fragment obtained with the primers SPI2a

(5'gctggctcaggtaacgccagaacaacgtgcgccggagtaagtgtaggctgga gctgcttc3') and SPI2b (5'tcaagcactgctctaacgctattaccctcttaac cttcgcatatgaatatcctccttag3'). SPI2a harbors 40 nucleotides that bind upstream of the $s s a G$ gene, and 20 nucleotides (in bold) that correspond to PS1. SPI2b harbors 40 nucleotides that bind at the end of the ssaU gene, and 20 nucleotides (in bold) that correspond to PS2. The deletions were verified by PCR from the genomic DNA using the appropriate primers. The $\Delta$ spi1 and $\Delta$ spi2 mutations were introduced into $\chi 4138$ by P22-mediated transduction to construct $\chi 9648$ and $\chi 9649$, respectively. $\chi 9650$ was constructed by transducing the $\Delta$ spi1 mutation into $\chi 9649$. All mutant strains were assayed for in vitro growth rate and were comparable to the wild type (data not shown), as well as tested for invasion in the macrophage cell line MQ-NSCU [31]. All strains containing the $\Delta$ spi1 mutation were approximately thirty times less invasive than those with an intact SPI1 (data not shown).

\section{Animal Infection}

All the animal experiments were conducted in accordance with protocols approved by the Arizona State University Institutional Animal Care and Use Committee. Specificpathogen-free fertile white leghorn eggs were obtained from SPAFAS Inc. (Roanoke, IL.) and hatched at the animal facilities of the Biodesign Institute, Arizona State University. At hatching, chicks were placed into isolators equipped with HEPA filters. The bacterial strains were grown to an $\mathrm{OD}_{600}$ of $\sim 0.8$. Equal volumes of cultures of strains that were co-administered were mixed and centrifuged at $4,000 \times \mathrm{g}$ at room temperature. The cells were then suspended in phosphate-buffered saline containing $0.01 \%$ gelatin to a final concentration of approximately 2 $\times 10^{10} \mathrm{CFU} / \mathrm{ml}$. Dilutions of this suspension were plated onto LB plates containing the appropriate antibiotics for the determination of the density and of the ratio of the strains from each mixture. For the infections, one-weekold chickens were deprived of food and water for $6 \mathrm{~h}$ prior to bacterial administration. $50 \mu \mathrm{l}$ of bacterial suspension corresponding approximately to $10^{9} \mathrm{CFU}$ were orally administered to chickens. Food and water were returned to the birds 30 minutes after infection.

Female six week old BALB/c mice (Charles River Laboratories, Wilmington, MA) were fasted for food and water for six hours before oral infection with $20 \mu \mathrm{l}$ of bacterial 
suspension ( $\sim 10^{9} \mathrm{CFU}$ ) prepared as described above. Food and drink were returned 30 minutes after infection. For intra-peritoneal infection mice were injected with 100 of bacterial suspension containing $10^{3}-10^{5} \mathrm{CFU}$.

\section{Organ processing}

All animals were euthanized by asphyxiation with $\mathrm{CO}_{2}$. The spleen and an approximately $3 \mathrm{~cm}$ piece of the cecal pouch (wall and content) were aseptically taken from each bird and homogenized (PowerGen 125 S1, Fischer Scientific, Pittsburgh, PA) in PBS. The spleen, or the spleen and a piece of the liver were recovered aseptically from each mouse and homogenized. Dilutions of these samples were plated onto McConkey-1\% lactose (MC) plates containing the appropriate antibiotics. Samples from animals infected with $\chi 4138$ and $\chi 9648, \chi 4138$ and $\chi 9649, \chi 4138$ and $\chi 9650$, and $\chi 9648$ and $\chi 9648$ were plated onto MC-Nal and MC-Nal-Cm, MC-Nal and MCNal-Km, MC-Nal and MC-Nal-Cm, and MC-Nal, MC-Nal$\mathrm{Cm}$ and $\mathrm{MC}-\mathrm{Nal}-\mathrm{Km}$ plates, respectively. The ratios of the strains recovered from the organs were determined by enumerating the colonies on the different plates and by patching colonies from MC-Nal plates onto plates containing the appropriate antibiotics.

\section{Competitive index and statistical analysis}

The competitive index is given by dividing the ratio of two strains from an organ divided by the same ratio in the suspension used for the infection. The geometric means of the CIs were determined and a Student's $t$-test was used to determine whether the logarithmically transformed ratios differed significantly from 0 . A $P$ value below 0.05 was considered statistically significant.

\section{Authors' contributions}

RCIII provided the idea for this study. YD designed the experiments and constructed the mutants. YD, KA, and $\mathrm{MM}$ performed the animal experiments. YD wrote the manuscript.

RCIII and KA revised the manuscript. All authors read and approved the final manuscript.

\section{Acknowledgements}

We thank Dr Kenneth Roland, Biodesign Institute, Arizona State University for fruitful discussion and critical reading of the manuscript and Patti Senechal for technical assistance. This work was supported by grant no. Al24533 from the National Institute of Health.

\section{References}

I. Bopp CA, Brenner FW, Wells JG: Escherichia, Shigella, and Salmonella. In Manual of clinical microbiology 7th edition. Edited by: Murray P, Baron EJ, Pfaller MA, Tenover F, Yolken R. Washington DC: ASM Press; 1999:459-474.

2. Tauxe RV, Pavia AT: Salmonellosis: nontyphoidal. In Bacterial infections of humans: epidemiology and control 3rd edition. Edited by: Evans AS, Brachman PS. New York, N.Y.: Plenum Medical Book Co; 1998:613-630.
3. Parry CM, Hien TT, Dougan G, White NJ, Farrar J]: Typhoid fever. N Engl J Med 2002, 347(22): 1770-1782.

4. Anonymous: Typhoid vaccines: WHO position paper. Weekly epidemiological record 2008, 83:49-60.

5. DuPont HL: The growing threat of foodborne bacterial enteropathogens of animal origin. Clin Infect Dis 2007, 45(I0): $|353-| 36 \mid$.

6. Mead PS, Slutsker L, Dietz V, McCaig LF, Bresee JS, Shapiro C, Griffin PM, Tauxe RV: Food-related illness and death in the United States. Emerg Infect Dis 1999, 5(5):607-625.

7. Thorns CJ: Bacterial food-borne zoonoses. Rev Sci Tech 2000, 19(I):226-239.

8. Babu US, Raybourne RB: Impact of dietary components on chicken immune system and Salmonella infection. Expert Rev Anti Infect Ther 2008, 6(I): 12I-135.

9. Barrow PA, Huggins MB, Lovell MA, Simpson JM: Observations on the pathogenesis of experimental Salmonella typhimurium infection in chickens. Res Vet Sci 1987, 42(2): 194-199.

10. Barrow PA, Simpson J, Lovell M: Intestinal colonisation in the chicken by food-poisoning salmonella serotypes; Microbial characteristics associated with faecal excretion. Avian Pathol 1988, 17(3):57|-588.

II. Withanage GS, Wigley P, Kaiser P, Mastroeni P, Brooks H, Powers C, Beal R, Barrow P, Maskell D, McConnell I: Cytokine and chemokine responses associated with clearance of a primary Salmonella enterica serovar Typhimurium infection in the chicken and in protective immunity to rechallenge. Infect Immun 2005, 73(8):5173-5182.

12. Haraga A, Ohlson MB, Miller SI: Salmonellae interplay with host cells. Nat Rev Microbiol 2008, 6(I):53-66.

13. Santos RL, Zhang S, Tsolis RM, Kingsley RA, Adams LG, Baumler AJ: Animal models of Salmonella infections: enteritis versus typhoid fever. Microbes Infect 200I, 3(14-I5): I335-I344.

14. Barthel M, Hapfelmeier S, Quintanilla-Martinez L, Kremer M, Rohde M, Hogardt M, Pfeffer K, Russmann H, Hardt WD: Pretreatment of mice with streptomycin provides a Salmonella enterica serovar Typhimurium colitis model that allows analysis of both pathogen and host. Infect Immun 2003, 7 I(5):2839-2858.

15. Galan JE: Salmonella interactions with host cells: type III secretion at work. Annu Rev Cell Dev Biol 200I, I 7:53-86.

16. Waterman SR, Holden DW: Functions and effectors of the Salmonella pathogenicity island 2 type III secretion system. Cell Microbiol 2003, 5(8):50 I-5II.

17. Coombes BK, Coburn BA, Potter AA, Gomis S, Mirakhur K, Li Y, Finlay BB: Analysis of the contribution of Salmonella pathogenicity islands $I$ and 2 to enteric disease progression using a novel bovine ileal loop model and a murine model of infectious enterocolitis. Infect Immun 2005, 73(I I):7|6I-7I69.

18. Hapfelmeier S, Ehrbar K, Stecher B, Barthel M, Kremer M, Hardt WD: Role of the Salmonella Pathogenicity Island I Effector Proteins SipA, SopB, SopE, and SopE2 in Salmonella enterica Subspecies I Serovar Typhimurium Colitis in StreptomycinPretreated Mice. Infect Immun 2004, 72(2):795-809.

19. Brawn LC, Hayward RD, Koronakis V: Salmonella SPII effector SipA persists after entry and cooperates with a SPI2 effector to regulate phagosome maturation and intracellular replication. Cell Host Microbe 2007, I(I):63-75.

20. Lawley TD, Chan K, Thompson LJ, Kim CC, Govoni GR, Monack DM: Genome-wide screen for Salmonella genes required for longterm systemic infection of the mouse. PLOS Pathog 2006, 2(2):el I.

2I. Steele-Mortimer O, Brumell JH, Knodler LA, Meresse S, Lopez A, Finlay $B B$ : The invasion-associated type III secretion system of Salmonella enterica serovar Typhimurium is necessary for intracellular proliferation and vacuole biogenesis in epithelial cells. Cell Microbiol 2002, 4(I):43-54.

22. Coburn B, Li Y, Owen D, Vallance BA, Finlay BB: Salmonella enterica serovar Typhimurium pathogenicity island 2 is necessary for complete virulence in a mouse model of infectious enterocolitis. Infect Immun 2005, 73(6):3219-3227.

23. Hapfelmeier S, Stecher B, Barthel M, Kremer M, Muller AJ, Heikenwalder M, Stallmach T, Hensel M, Pfeffer K, Akira S, Hardt WD: The Salmonella pathogenicity island (SPI)-2 and SPI-I type III secretion systems allow Salmonella serovar typhimurium to trigger colitis via MyD88-dependent and MyD88-independent mechanisms. J Immunol 2005, 174(3): 1675-1685. 
24. Thijs IM, De Keersmaecker SC, Fadda A, Engelen K, Zhao H, McClelland M, Marchal K, Vanderleyden J: Delineation of the Salmonella enterica serovar Typhimurium HilA regulon through genome-wide location and transcript analysis. J Bacteriol 2007, 189(13):4587-4596.

25. Bustamante VH, Martinez LC, Santana FJ, Knodler LA, Steele-Mortimer O, Puente JL: HilD-mediated transcriptional cross-talk between SPI-I and SPI-2. Proc Nat Acad of Sci USA 2008, I05(38): |459|-|4596.

26. Porter SB, Curtiss R III: Effect of inv mutations on Salmonella virulence and colonization in I-day-old White Leghorn chicks. Avian Dis 1997, 4 I (I):45-57.

27. Jones MA, Hulme SD, Barrow PA, Wigley P: The Salmonella pathogenicity island $I$ and Salmonella pathogenicity island 2 type III secretion systems play a major role in pathogenesis of systemic disease and gastrointestinal tract colonization of Salmonella enterica serovar Typhimurium in the chicken. Avian Pathol 2007, 36(3): 199-203.

28. Turner AK, Lovell MA, Hulme SD, Zhang-Barber L, Barrow PA: Identification of Salmonella typhimurium genes required for colonization of the chicken alimentary tract and for virulence in newly hatched chicks. Infect Immun 1998, 66(5):2099-2106.

29. Morgan E, Campbell JD, Rowe SC, Bispham J, Stevens MP, Bowen AJ, Barrow PA, Maskell DJ, Wallis TS: Identification of host-specific colonization factors of Salmonella enterica serovar Typhimurium. Mol Microbiol 2004, 54(4):994-10I0.

30. Beuzon CR, Holden DW: Use of mixed infections with Salmonella strains to study virulence genes and their interactions in vivo. Microbes Infect 200I, 3(14-15): 1345-1352.

31. Qureshi MA, Miller L, Lillehoj HS, Ficken MD: Establishment and characterization of a chicken mononuclear cell line. Vet Immunol Immunopathol I990, 26(3):237-250.

32. Parsons DA, Heffron F: sciS, an icmF homolog in Salmonella enterica serovar Typhimurium, limits intracellular replication and decreases virulence. Infect Immun 2005, 73(7):4338-4345.

33. Murray RA, Lee CA: Invasion genes are not required for Salmonella enterica serovar typhimurium to breach the intestinal epithelium: evidence that salmonella pathogenicity island has alternative functions during infection. Infect Immun 2000 , 68(9):5050-5055.

34. Bohez L, Gantois I, Ducatelle R, Pasmans F, Dewulf J, Haesebrouck F, Van Immerseel F: The Salmonella Pathogenicity Island 2 regulator ssrA promotes reproductive tract but not intestinal colonization in chickens. Vet Microbiol 2008, I 26(I-3):216-224.

35. Zhang X, Kelly SM, Bollen W, Curtiss R III: Protection and immune responses induced by attenuated Salmonella typhimurium UK-I strains. Micro Pathog |999, 26(3): |2|-|30.

36. Curtiss R 3rd, Porter SB, Munson M, Tinge SA, Hassan JO, GentryWeeks C, Kelly SM: Nonrecombinant and recombinant avirulent Salmonella live vaccines for poultry. In Colonization control of human bacterial enteropathogens in poultry Edited by: Edited by Blankenship LC, Bailey JS, Cox NA, Stern NJ, Meinersmann RJ. I99I: I69198.. New York, N.Y.: Academic Press; 1991:169-198.

37. Wigley P, Jones MA, Barrow PA: Salmonella enterica serovar Pullorum requires the Salmonella pathogenicity island 2 type III secretion system for virulence and carriage in the chicken. Avian Pathol 2002, 3 I (5):50I-506.

38. Jones MA, Wigley P, Page KL, Hulme SD, Barrow PA: Salmonella enterica serovar Gallinarum requires the Salmonella pathogenicity island 2 type III secretion system but not the Salmonella pathogenicity island I type III secretion system for virulence in chickens. Infect Immun 200I, 69(9):547I-5476.

39. Boyen F, Pasmans F, Van Immerseel F, Morgan E, Botteldoorn N, Hey ndrickx M, Volf J, Favoreel H, Hernalsteens JP, Ducatelle R, Haesebrouck F: A limited role for SsrA/B in persistent Salmonella Typhimurium infections in pigs. Vet Microbiol 2008, I28(34):364-373.

40. Bohez L, Ducatelle R, Pasmans F, Botteldoorn N, Haesebrouck F, Van Immerseel F: Salmonella enterica serovar Enteritidis colonization of the chicken caecum requires the HilA regulatory protein. Vet Microbiol 2006, I I 6(I-3):202-2 I0.

4I. Bertani G: Studies on lysogenesis. I. The mode of phage liberation by lysogenic Escherichia coli. J Bacteriol I951, 62:293-300.
42. Sambrook J, Fritsch EF, Maniatis T: Molecular Cloning: a laboratory manual. second edition. Cold Spring Harbor, N.Y.: Cold Spring Harbor Laboratory Press; 1989.

43. Maloy SR, Stewart V], Taylor RK: Genetic analysis of pathogenic bacteria: a laboratory manual. Cold Spring Harbor, N.Y.: Cold Spring Harbor Laboratory Press; 1996.

44. Gulig PA, Curtiss R III: Plasmid-associated virulence of Salmonella typhimurium. Infect Immun I 987, 55( I 2):289I-290I

45. Merighi M, Ellermeier CD, Slauch JM, Gunn JS: Resolvase-in vivo expression technology analysis of the Salmonella enterica serovar Typhimurium PhoP and PmrA regulons in BALB/c mice. J Bacteriol 2005, I87(2 I):7407-74l6.

46. Datsenko KA, Wanner BL: One-step inactivation of chromosomal genes in Escherichia coli K- 12 using PCR products. Proc Nat Acad Sci USA 2000, 97( 1 2):6640-6645.
Publish with Biomed Central and every scientist can read your work free of charge

"BioMed Central will be the most significant development for disseminating the results of biomedical research in our lifetime. "

Sir Paul Nurse, Cancer Research UK

Your research papers will be:

- available free of charge to the entire biomedical community

- peer reviewed and published immediately upon acceptance

- cited in PubMed and archived on PubMed Central

- yours - you keep the copyright
BioMedcentral 